Ann. Génét. Sél. anim., I969, 1 (I), 39-47.

\title{
VARIANCE ET COVARIANCE GÉNOTYPIQUES $A$ PRIORI DANS LE CAS D'UNE POPULATION DE TÉTRAPLOÏDES SOUMISE A L'AUTOFÉCONDATION
}

\author{
Jacqueline BOUFFETTE et A.-R. BOUFFETTE \\ Université de Lyon, \\ Faculté de Sciences-Mathématiques, \\ 43, boulevard du II Novembre 1918 , \\ 69 - Villeurbanne
}

\section{SOMMAIRE}

Après avoir rappelé les résultats permettant le calcul de la variance génotypique, nous montrons comment établir les expressions des coefficients d'identité restreinte d'ordre 8 et de rang 2. A partir de là, nous donnons l'expression de la covariance génotypique entre 2 zygotes tétraploïdes issus d'un même individu.

\section{INTRODUCTION}

Jacqueline BOUFFETTE (1966) a décomposé la variance génotypique d'un zygote tétraploïde et la covariance génotypique entre 2 zygotes tétraploïdes quelconques en fonction de toutes les interactions alléliques. Cette décomposition a été possible grâce à la notion de situation d'identité introduite par GrLLors (I965) lorsqu'il généralisa les travaux de MALEcot (r948) sur les diploïdes.

Dire que deux gènes homologues sont identiques, c'est affirmer qu'ils dérivent par descendance mendélienne, sans mutation, d'un même gène ancêtre. Cette relation d'identité étant une relation d'équivalence, permet de définir sur tout ensemble de gènes homologues, un ensemble-quotient, appelé situation d'identité restreinte, formé de classes de gènes identiques.

Si l'on considère quatre gènes homologues $i, j, k, l$, non discernables, présents chez un zygote tétraploïde et supposés être les seules causes d'un caractère quantitatif étudié, il est possible de définir 5 situations d'identité restreinte. 
A chaque situation d'identité restreinte est attaché un coefficient d'identité restreinte qui est la probabilité de réalisation de cette situation.

Ainsi, les probabilités des situations $(i \equiv j \equiv k \equiv l), \quad(i \equiv j \equiv k \neq l)$, $(i \equiv j \not \equiv k \equiv l),(i \equiv j \not \equiv k \not \equiv l), \quad(i \not \equiv j \not \equiv k \not \equiv l)$ sont respectivement $\Delta_{1}, \Delta_{\mathbf{2}}$, $\Delta_{3}, \Delta_{4}$ et $\Delta_{5}$. On a noté $i \equiv j$ lorsque les 2 gènes $i$ et $j$ sont identiques et $i \neq j$ lorsqu'ils sont non identiques.

Pour un zygote tétraploïde pris à la génération $n$, dans une population soumise à l'autofécondation, A.-R. BoufFetite (I966) a déterminé les coefficients d'identité restreinte $\Delta_{k}$ d'ordre 4 et de rang $\mathbf{x}$.

TABLEAU I

Coefficients d'identité restreinte d'ordre 4 et de rang $\mathrm{I}$

$$
\begin{aligned}
& \Delta_{1}(n)=\mathrm{I}+b\left(\frac{\mathrm{I}}{6} \mathrm{Q}^{2}\right)^{n}-c\left(\frac{3}{8} \mathrm{P}^{2}+\frac{5}{4} \mathrm{PQ}+\frac{7}{\mathrm{I} 2} \mathrm{Q}^{2}\right)\left(\frac{\mathrm{r}}{2} \mathrm{P}+\frac{5}{6} \mathrm{Q}\right)^{n}+d\left(\frac{\mathrm{I}}{2} \mathrm{Q}\right)^{n} \\
& \Delta_{2}(n)=-4 b\left(\frac{\mathrm{I}}{6} \mathrm{Q}^{2}\right)^{n}+c\left(\mathrm{PQ}+\frac{\mathrm{I}}{3} \mathrm{Q}^{2}\right)\left(\frac{\mathrm{I}}{2} \mathrm{P}+\frac{5}{6} \mathrm{Q}\right)^{n}-2 d\left(\frac{\mathrm{I}}{2} \mathrm{Q}\right)^{n} \\
& \Delta_{3}(n)=(3 b+e)\left(\frac{\mathrm{I}}{6} \mathrm{Q}^{2}\right)^{n}+c\left(\frac{3}{8} \mathrm{P}^{2}+\frac{\mathrm{I}}{4} \mathrm{PQ}+\frac{\mathrm{r}}{4} \mathrm{Q}^{2}\right)\left(\frac{\mathrm{I}}{2} \mathrm{P}+\frac{5}{6} \mathrm{Q}\right)^{n}-d\left(\frac{\mathrm{I}}{2} \mathrm{Q}\right)^{n} \\
& \Delta_{4}(n)=-2 e\left(\frac{\mathrm{I}}{6} \mathrm{Q}^{2}\right)^{n}+2 d\left(\frac{\mathrm{I}}{2} \mathrm{Q}\right)^{n} \\
& \Delta_{5}(n)=e\left(\frac{\mathrm{I}}{6} \mathrm{Q}^{2}\right)^{n}
\end{aligned}
$$

Dans ces expressions, $P$ désijne la probabilité qu'un gamète quelconque porte 2 gènes frères, c'est-à-dire copies d'un même gène présent chez l'individu qui lui donne naissance. De plus, on a posé $\mathrm{P}+\mathrm{Q}=\mathbf{I} . b, c, d, e$ sont des constantes qui ne dépendent que de la situation d'identité dans laquelle se trouve le zygote de la génération origine. Ainsi, si les 4 gènes du zygote de la génération zéro sont tous non identiques, on obtient :

$$
b=-\frac{3 \mathrm{P}^{2}+2 \mathrm{PQ}+2 \mathrm{Q}^{2}}{2\left(4-\mathrm{P}^{2}\right)} \quad c=\frac{\mathrm{I} 2}{4-\mathrm{P}^{2}} \quad d=e=\mathrm{I}
$$

On se propose maintenant de donner l'expression de la variance génotypique, puis de déterminer les 66 coefficients d'identité restreinte d'ordre 8 , de rang 2 définis par Jacqueline BoufFETrE (I966) pour 2 zygotes pris à la même génération $n+\mathrm{I}$. Ces coefficients nous permettront de déterminer la covariance génotypique a priori.

\section{EXPRESSION DE LA VARIANCE}

Lorsqu'on admet que les variables aléatoires zygotiques attachées à 2 gènes non identiques sont stochastiquement indépendantes, il est possible alors d'exprimer la variance de la variable génotypique $\widehat{Z}$, décomposée sous la forme :

$$
\begin{gathered}
\widehat{Z}=Z_{1}+Z_{2}+Z_{3}+Z_{4}+D_{12}+D_{13}+D_{14}+D_{23}+D_{24}+D_{34}+D_{123}+D_{134} \\
+D_{124}+D_{234}+D_{1234}
\end{gathered}
$$


A l'aide de la formule donnée par Jacqueline BoufFetTte (I966) dans laquelle on a posé $\Delta_{k}(n)=\Delta_{k}$, on obtient la formule I suivante :

$$
\begin{aligned}
& \operatorname{var}\left(\overline{Z)}=\left[4 \Delta_{5}+6 \Delta_{4}+8 \Delta_{3}+\text { Io } \Delta_{2}+\text { I6 } \Delta_{1}\right] \mathrm{E}\left(Z_{i}^{2}\right)\right. \\
& +\left[4 \Delta_{4}+3 \Delta_{3}+\mathrm{I} 8 \Delta_{2}+48 \Delta_{1}\right] \mathrm{E}\left(Z_{i} D_{i i}\right)+\left[6 \Delta_{2}+32 \Delta_{1}\right] \\
& \mathrm{E}\left(Z_{i} \mathrm{D}_{i i i}\right)+\left[4 \Delta_{4}+\mathrm{I} 6 \Delta_{3}+6 \Delta_{2}\right] \mathrm{E}\left(Z_{i} \mathrm{D}_{i j j}\right) \\
& +8 \Delta_{1} \cdot \mathrm{E}\left(Z_{i} \mathrm{D}_{i i i}\right)+8 \Delta_{3} \cdot \mathrm{E}\left(Z_{i} \mathrm{D}_{i i j j}\right)+2 \Delta_{2} \cdot \mathrm{E}\left(\mathrm{Z}_{i} \mathrm{D}_{i j j j}\right) \\
& +\left[\Delta_{4}+2 \Delta_{3}+9 \Delta_{2}+36 \Delta_{1}\right] . \mathrm{E}\left(\mathrm{D}_{i i}^{2}\right) \\
& +\left[6 \Delta_{5}+9 \Delta_{4}+\mathrm{I} 6 \Delta_{3}+9 \Delta_{2}\right] \mathrm{E}\left(\mathrm{D}_{l j}^{2}\right)+\left[2 \Delta_{3}-36\left(f^{(2)}\right)^{2}\right] \\
& {\left[\mathrm{E}\left(\mathrm{D}_{i i}\right)\right]^{2}+\left[6 \Delta_{2}+4^{8} \Delta_{1}\right] \cdot \mathrm{E}\left(\mathrm{D}_{i i} \mathrm{D}_{i i i}\right)-48 f^{(2)} . f^{(3)} \text {. }} \\
& \mathrm{E}\left(\mathrm{D}_{i i}\right) \cdot \mathrm{E}\left(\mathrm{D}_{i l i}\right)+8 \Delta_{3} \cdot \mathrm{E}\left(\mathrm{D}_{i l} \mathrm{D}_{i j j}\right)+\left[8 \Delta_{4}+32 \Delta_{3}+\mathrm{I} 8 \Delta_{2}\right] \\
& \mathrm{E}\left(\mathrm{D}_{i j} \mathrm{D}_{i i j}\right)+\mathrm{I} 2 \Delta_{1} \cdot \mathrm{E}\left(\mathrm{D}_{i l} \mathrm{D}_{i t i i}\right)-\mathrm{I} 2 f^{(2)} \cdot f^{(4)} \mathrm{E}\left(\mathrm{D}_{i i}\right) \text {. } \\
& \mathrm{E}\left(\mathrm{D}_{i t i}\right)-\mathrm{I} 2 f^{(2)} \cdot f^{(2,2)} \mathrm{E}\left(\mathrm{D}_{i i}\right) \cdot \mathrm{E}\left(\mathrm{D}_{i t j j}\right)+4 \Delta_{3} \text {. } \\
& \mathrm{E}\left(\mathrm{D}_{i l} \mathrm{D}_{i i j j}\right)+6 \Delta_{2} \cdot \mathrm{E}\left(\mathrm{D}_{i j} \mathrm{D}_{i i i j}\right)+8 \Delta_{3} \cdot \mathrm{E}\left(\mathrm{D}_{i j} \mathrm{D}_{i t j j}\right) \\
& +2 \Delta_{4} \cdot \mathrm{E}\left(\mathrm{D}_{i j} \mathrm{D}_{i j k k}\right)+\left[\Delta_{2}+\mathrm{I} 6 \Delta_{1}\right] \cdot \mathrm{E}\left(\mathrm{D}_{i j k}^{2}\right)+ \\
& +\left[2 \Delta_{4}+8 \Delta_{3}+9 \Delta_{2}\right] \cdot \mathrm{E}\left(\mathrm{D}_{i i j}^{2}\right)+\left[4 \Delta_{5}+4 \Delta_{4}\right] \cdot \mathrm{E}\left(\mathrm{D}_{i j k}^{2}\right) \\
& -\mathrm{I} 6\left[f^{(3)}\right]^{2}\left[\mathrm{E}\left(\mathrm{D}_{i i i}\right)\right]^{2}+8 \Delta_{3} \cdot \mathrm{E}\left(\mathrm{D}_{i i j} \mathrm{D}_{i j j}\right) \\
& +8 \Delta_{1} \cdot \mathrm{E}\left(\mathrm{D}_{i t i} \mathrm{D}_{i t i}\right)-8 f^{(3)} \cdot f^{(4)} \cdot \mathrm{E}\left(\mathrm{D}_{i i i}\right) \cdot \mathrm{E}\left(\mathrm{D}_{i t i}\right) \\
& -8 f^{(3)} \cdot f^{(2,2)} \cdot \mathrm{E}\left(\mathrm{D}_{i i l}\right) \cdot \mathrm{E}\left(\mathrm{D}_{i i j j}\right)+6 \Delta_{2} \mathrm{E}\left(\mathrm{D}_{i i j} \mathrm{D}_{i t i j}\right) \\
& +4 \Delta_{4} \cdot \mathrm{E}\left(\mathrm{D}_{i j k} \mathrm{D}_{i i j k}\right)+8 \Delta_{3} \cdot \mathrm{E}\left(\mathrm{D}_{i l j} \mathrm{D}_{i l j j}\right)- \\
& -\left[f^{(2,2)}\right]^{2}\left[\mathrm{E}\left(\mathrm{D}_{i l j j}\right)\right]^{2}+\Delta_{5} \cdot \mathrm{E}\left(\mathrm{D}_{i j k l}^{2}\right)-2 f^{(2,2)} \cdot f^{(4)} \text {. } \\
& \mathrm{E}\left(\mathrm{D}_{i t i i}\right) \cdot \mathrm{E}\left(\mathrm{D}_{i t j j}\right)+\Delta_{4} \mathrm{E}\left(\mathrm{D}_{i j k k}^{2}\right)-\left(f^{(4)}\right)^{2}\left[\mathrm{E}\left(\mathrm{D}_{i t i l}\right)\right]^{2} \\
& +\Delta_{3} \cdot \mathrm{E}\left(\mathrm{D}_{i i j j}^{2}\right)+\Delta_{2} \cdot \mathrm{E}\left(\mathrm{D}_{i i i j}^{2}\right)+\Delta_{1} \cdot \mathrm{E}\left(\mathrm{D}_{i i i i}^{2}\right)
\end{aligned}
$$

où $f^{(r, s)}$ désigne la probabilité pour que, $r+s=t$ gènes homologues étant arbitrairement choisis dans un zygote, soient répartis en classes d'identité de $r$ et $s$ gènes respectivement.

Les variables aléatoires de dominance introduites font intervenir 2,3 ou 4 gènes. Pour préciser la situation d'identité restreinte dans laquelle ils se trouvent, on a noté d'un même indice des gènes identiques. Par exemple, $\mathrm{D}_{t i j}$ désigne la dominance existant entre 2 gènes identiques et un troisième gène non identique aux deux premiers. Notre notation des variables aléatoires génétiques est ainsi fondamentalement différente de la notation classique de KEMPTHORNE (I957) et de Galiais (r967), dans laquelle deux mêmes indices désignent des gènes isoactifs (allèles).

Quand le nombre de générations augmente, il est facile de voir que la variance génotypique tend vers une limite qui est la variance génotypique d'un zygote ayant ses 4 gènes identiques. Ce résultat rejoint le fait connu, qu'en absence de mutations et de sélection, la consanguinité augmente avec le temps. Ainsi, diploïdes et tétraploïdes ont même comportement asymptotique.

\section{EXPRESSION DE LA COVARIANCE ENTRE 《 PLEINS FRÈRES "}

L'expression de la covariance génotypique entre 2 zygotes quelconques fait intervenir les probabilités $P_{k}$ des 66 situations d'identité restreinte d'ordre 8 et de rang 2 définies par Jacqueline BoufFetre (Ig66). 
Lorsque les 2 zygotes $\mathrm{I}$ et $\mathrm{J}$ appartiennent à la même génération $n+\mathrm{I}$, ceux-ci descendant d'un même individu $\mathrm{H}_{n}$ de la génération $n$, on peut exprimer les différentes probabilités $\mathrm{P}_{k}(n+\mathrm{I})$ comme combinaisons linéaires des probabilités connues $\Delta_{i}(n)$ :

$$
\mathrm{P}_{k}(n+\mathrm{I})=\sum_{i=\mathrm{I}}^{i=5} a_{i} \Delta_{i}(n) \text { pour } n \geqslant 0
$$

Les coefficients $a_{i}$ sont des constantes qui s'expriment à 1'aide de la probabilité $\mathrm{P}$ déjà définie.

Il est évident que certaines situations d'identité d'ordre 8 et de rang 2 ont une probabilité nulle de se réaliser. Par exemple, il n'est pas possible que les 8 gènes obtenus soient non identiques, donc $\mathrm{P}_{1}(n+\mathrm{I})=0$. Il en est d'ailleurs de même pour toutes les situations formées de plus de 4 classes d'identité.

Calculons $\mathrm{P}_{12}(n+\mathrm{I})$, première probabilité non nulle dans la classification des 66 situations d'identité d'ordre 8 et de rang 2 de Jacqueline Boufretre (r966). C'est la probabilité d'avoir 2 zygotes I et $\mathrm{J}$ non parents et formés chacun de 2 paires de gènes identiques, c'est-à-dire d'obtenir 2 zygotes dans la situation

$\left(\begin{array}{l|l}2 & 0 \\ 2 & 0 \\ 0 & 2 \\ 0 & 2\end{array}\right)$ que nous noterons plus simplement. (I2).

Supposons $\mathrm{H}_{n}$ formé de gènes non identiques, événement de probabilité $\Delta_{5}(n)$.

Si I et $\mathrm{J}$ sont formés par la fusion de gamètes portant des gènes non frères (événement de probabilité $Q^{4}$ ), ils sont alors dans la situation (I2) avec la probabilité I $\times \frac{\mathrm{I}}{6} \times \frac{\mathrm{I}}{6} \times \frac{\mathrm{I}}{6}=\frac{\mathrm{I}}{2 \mathrm{I} 6}$.

Si I et J sont formés par la fusion de gamètes portant des gènes frères (événement de probabilité $\mathrm{P}^{4}$ ), ils sont alors dans la situation (I2) avec la probabilité I $\times \frac{3}{4} \times \frac{I}{2} \times \frac{I}{4}=\frac{3}{32}$.

Si I (ou $J$ ) est formé par la fusion de gamètes portant des gènes frères et $J$ (ou I) est formé par la fusion de gamètes portant des gènes non frères (événement de probabilité $\mathrm{P}^{2} \mathrm{Q}^{2}$ ), ils sont alors dans la situation (I2) avec la probabilité $\mathrm{I} \times \frac{3}{4} \times \frac{\mathrm{I}}{6} \times \frac{\mathrm{I}}{6}=\frac{\mathrm{I}}{48}$.

Si $\mathrm{H}_{n}$ possède au moins 2 gènes identiques, alors $\mathrm{I}$ et $\mathrm{J}$ ne peuvent être dans la situation ( $\mathrm{x} 2$ ), car celle-ci implique 4 gènes non identiques dans $\mathrm{H}_{n}$, d'où :

$$
\mathrm{P}_{12}(n+\mathrm{I})=\left(\frac{\mathrm{I}}{2 \mathrm{I} 6} \mathrm{Q}^{4}+\frac{3}{32} \mathrm{P}^{4}+\frac{\mathrm{I}}{48} 2 \mathrm{P}^{2} \mathrm{Q}^{2}\right) \Delta_{5}(n)
$$


En utilisant le même mode de raisonnement que ci-dessus, nous obtenons les expressions des $\mathrm{P}_{k}(n+\mathrm{I})$ non nuls suivantes :

\section{TABLEAU 2}

Coefficients d'identité restreinte d'ordre 8 et de rang 2

$$
\begin{aligned}
& \mathrm{P}_{12}(n+\mathrm{I})=\Delta_{5}(n) \cdot\left[\frac{\mathrm{Q}_{4}}{2 \mathrm{I} 6}+\frac{3^{\mathrm{P}^{4}}}{3^{2}}+\frac{\mathrm{P}^{2} \mathrm{Q}^{2}}{24}\right] \\
& \mathrm{P}_{13}(n+\mathrm{I})=\Delta_{5}(n) \cdot\left[\frac{\mathrm{Q}^{4}}{36}\right] \\
& \mathbf{P}_{14}(n+\mathrm{I})=\Delta_{5}(n) \cdot\left[\frac{\mathrm{Q}_{4}}{27}+\frac{\mathrm{PQ}^{3}}{9}+\frac{\mathrm{P}^{2} \mathrm{Q}^{2}}{\mathrm{I} 2}\right] \\
& \mathrm{P}_{22}(n+\mathrm{I})=\Delta_{5}(n) \cdot\left[\frac{\mathrm{P}^{3} \mathrm{Q}}{4}+\frac{\mathrm{PQ}^{3}}{\mathrm{I} 8}\right] \\
& \mathrm{P}_{23}(n+\mathrm{I})=\Delta_{5}(n) \cdot\left[\frac{2 \mathrm{Q}^{4}}{27}+\frac{\mathrm{P}^{2} \mathrm{Q}^{2}}{3}+\frac{\mathrm{P}^{3} \mathrm{Q}}{2}+\frac{\mathrm{PQ}^{3}}{9}\right] \\
& \mathrm{P}_{24}(n+\mathrm{I})=\Delta_{5}(n) \cdot\left[\frac{2 \mathrm{PQ}^{3}}{9}+\frac{\mathrm{P}^{2} \mathrm{Q}^{2}}{3}\right] \\
& \mathrm{P}_{25}(n+\mathrm{r})=\Delta_{5}(n) \cdot\left[\frac{2 \mathrm{Q}^{4}}{9}+\frac{\mathrm{PQ}^{3}}{3}\right] \\
& \mathrm{P}_{26}(n+\mathrm{I})=\Delta_{5}(n) \cdot\left[\frac{4 \mathrm{Q}^{4}}{27}+\frac{4^{\mathrm{P} Q^{3}}}{9}+\frac{\mathrm{P}^{2} Q^{2}}{3}\right] \\
& \mathrm{P}_{27}(n+\mathrm{I})=\Delta_{5}(n) \cdot\left[\frac{\mathrm{P}^{2} \mathrm{Q}^{2}}{6}\right] \\
& \mathrm{P}_{28}(n+\mathrm{I})=\Delta_{5}(n) \cdot\left[\frac{\mathrm{Q}_{4}}{\mathrm{I} 8}+\frac{\mathrm{P}^{2} \mathrm{Q}^{2}}{4}\right] \\
& \mathrm{P}_{28}(n+\mathrm{r})=\Delta_{5}(n) \cdot\left[\frac{\mathrm{PQ}^{3}}{9}+\frac{\mathrm{P}^{2} \mathrm{Q}^{2}}{6}\right] \\
& \mathrm{P}_{30}(n+\mathrm{I})=\Delta_{5}(n) \cdot\left[\frac{2 \mathrm{Q}^{4}}{27}+\frac{2 \mathrm{PQ}^{3}}{9}+\frac{\mathrm{P}^{2} \mathrm{Q}^{2}}{6}\right] \\
& \mathrm{P}_{31}(n+\mathrm{I})=\Delta_{5}(n) \cdot\left[\frac{\mathrm{P}^{2} \mathrm{Q}^{2}}{6}\right]+\Delta_{4}(n) \cdot\left[\frac{\mathrm{PQ}^{3}}{27}+\frac{\mathrm{P}^{2} Q^{2}}{6}\right] \\
& \mathrm{P}_{32}(n+\mathrm{x})=\Delta_{5}(n) \cdot\left[\frac{\mathrm{I}}{2} \mathrm{P}^{3} \mathrm{Q}+\frac{\mathrm{I}}{9} \mathrm{PQ}^{3}\right]+\Delta_{4}(n) \cdot\left[\frac{\mathrm{r}}{8 \mathrm{I}} \mathrm{Q}^{4}+\frac{5}{36} \mathrm{P}^{2} \mathrm{Q}^{2}+\frac{5}{\mathrm{I} 2} \mathrm{P}^{3} \mathrm{Q}+\frac{4}{27} \mathrm{PQ}^{3}\right] \\
& \mathrm{P}_{33}(n+\mathrm{r})=\Delta_{5}(n) \cdot\left[\frac{\mathrm{I}}{27} \mathrm{Q}^{4}+\frac{\mathrm{I}}{6} \mathrm{P}^{2} \mathrm{Q}^{2}+\frac{\mathrm{T}}{4} \mathrm{P}^{3} \mathrm{Q}+\frac{\mathrm{I}}{\mathrm{I} 8} \mathrm{PQ}^{3}\right]+ \\
& +\Delta_{4}(n) \cdot\left[\frac{7}{108} Q^{4}+\frac{17}{72} P^{2} Q^{2}+\frac{5}{24} P^{3} Q+\frac{13}{108} P^{3}\right] \\
& \mathbf{P}_{34}(n+\mathrm{I})=\Delta_{5}(n) \cdot\left[\frac{2}{27} Q^{4}+\frac{2}{9} \mathrm{PQ}^{3}+\frac{\mathrm{I}}{6} \mathrm{P}^{2} \mathrm{Q}^{2}\right]+\Delta_{4}(n) \cdot\left[\frac{4}{27} \mathrm{Q}^{4}+\frac{\mathrm{I}}{3} \mathrm{PQ}^{3}+\frac{\mathrm{I}}{6} \mathrm{P}^{2} \mathrm{Q}^{2}\right] \\
& \mathrm{P}_{38}(n+\mathrm{I})=\Delta_{5}(n) \cdot\left[\frac{\mathrm{r}}{\mathrm{I} 2} \mathrm{P}^{2} \mathrm{Q}^{2}+\frac{\mathrm{I}}{8} \mathrm{P}^{3} \mathrm{Q}\right] \\
& \mathrm{P}_{39}(n+\mathrm{I})=\Delta_{5}(n) \cdot\left[\frac{\mathrm{I}}{27} \mathrm{Q}^{4}+\frac{\mathrm{I}}{6} \mathrm{P}^{2} \mathrm{Q}^{2}+\frac{\mathrm{T}}{4} \mathrm{P}^{3} \mathrm{Q}+\frac{\mathrm{T}}{\mathrm{I} 8} \mathrm{PQ}^{3}\right] \\
& \mathrm{P}_{40}(n+\mathrm{I})=\Delta_{5}(n) \cdot\left[\frac{2}{9} \mathrm{PQ}^{3}+\frac{\mathrm{r}}{3} \mathrm{P}^{2} \mathrm{Q}^{2}\right] \\
& \mathrm{P}_{41}(n+\mathrm{I})=\Delta_{5}(n) \cdot\left[\frac{2}{27} \mathrm{Q}^{4}+\frac{2}{9} \mathrm{PQ}^{3}+\frac{\mathrm{I}}{6} \mathrm{P}^{2} \mathrm{Q}^{2}\right] \\
& \mathrm{P}_{42}(n+\mathrm{I})=\Delta_{5}(n) \cdot\left[\frac{\mathrm{x}}{3} \mathrm{PQ}^{3}\right]
\end{aligned}
$$




$$
\begin{aligned}
& \mathrm{P}_{43}(n+\mathrm{I})=\Delta_{5}(n) \cdot\left[\frac{3}{\mathrm{r} 6} \mathrm{P}^{4}+\frac{\mathrm{I}}{24} \mathrm{P}^{2} \mathrm{Q}^{2}\right]+\Delta_{4}(n) \cdot\left[\frac{\mathrm{I}}{648} \mathrm{Q}^{4}+\frac{\mathrm{I}}{8} \mathrm{P}^{4}+\frac{7}{\mathrm{I} 44} \mathrm{P}^{2} \mathrm{Q}^{2}+\frac{\mathrm{I}}{\mathrm{I} 6} \mathrm{P}^{3} \mathrm{Q}+\frac{\mathrm{I}}{\mathrm{IO} 8} \mathrm{PQ}^{3}\right] \\
& \mathrm{P}_{44}(n+\mathrm{I})=\Delta_{5}(n) \cdot\left[\frac{\mathrm{I}}{54} \mathrm{Q}^{4}+\frac{3}{8} \mathrm{P}^{4}+\frac{\mathrm{I}}{6} \mathrm{P}^{2} \mathrm{Q}^{2}\right]+\Delta_{4}(n) \cdot\left[\frac{\mathrm{I}}{27} \mathrm{Q}^{4}+\frac{\mathrm{I}}{4} \mathrm{P}^{4}+\frac{5}{24} \mathrm{P}^{2} \mathrm{Q}^{2}\right. \\
& \left.+\frac{\mathrm{I}}{8} \mathrm{P}^{3} \mathrm{Q}+\frac{5}{\mathrm{IO} 8} \mathrm{PQ}^{3}\right] \\
& \mathrm{P}_{45}(n+\mathrm{I})=\Delta_{5}(n) \cdot\left[\frac{2}{9} \mathrm{PQ}^{3}+\frac{\mathrm{I}}{3} \mathrm{P}^{2} \mathrm{Q}^{2}\right]+\Delta_{4}(n) \cdot\left[\frac{4}{8 \mathrm{I}} \mathrm{Q}^{4}+\frac{7}{\mathrm{I} 8} \mathrm{PQ}^{3}+\frac{7}{\mathrm{I} 8} \mathrm{P}^{2} \mathrm{Q}^{2}\right] \\
& \mathrm{P}_{48}(n+\mathrm{I})=\Delta_{5}(n) \cdot\left[\frac{\mathrm{I}}{27} \mathrm{Q}^{4}+\frac{\mathrm{I}}{9} \mathrm{PQ}^{3}+\frac{\mathrm{I}}{\mathrm{I} 2} \mathrm{P}^{2} \mathrm{Q}^{2}\right]+\Delta_{4}(n) \cdot\left[\frac{\mathrm{II}}{\mathrm{IO} 8} \mathrm{Q}^{4}+\frac{\mathrm{I}}{6} \mathrm{PQ}^{3}+\frac{\mathrm{I}}{\mathrm{I} 2} \mathrm{P}^{2} \mathrm{Q}^{2}\right] \\
& \mathrm{P}_{47}(n+\mathrm{I})=\Delta_{5}(n) \cdot\left[\frac{\mathrm{I}}{4} \mathrm{P}^{3} \mathrm{Q}\right]+\Delta_{4}(n) \cdot\left[\frac{\mathrm{I}}{\mathrm{I} 2} \mathrm{P}^{2} \mathrm{Q}^{2}+\frac{5}{24} \mathrm{P}^{3} \mathrm{Q}+\frac{\mathrm{I}}{\mathrm{IO} 8} \mathrm{PQ}^{3}\right] \\
& \mathrm{P}_{48}(n+\mathrm{I})=\Delta_{5}(n) \cdot\left[\frac{5}{27} \mathrm{Q}^{4}+\frac{\mathrm{I}}{3} \mathrm{P}^{2} \mathrm{Q}^{2}+\frac{\mathrm{I}}{2} \mathrm{P}^{3} \mathrm{Q}+\frac{\mathrm{I}}{9} \mathrm{PQ}^{3}\right]+ \\
& +\Delta_{4}(n) \cdot\left[\frac{\mathrm{II}}{\mathrm{IO} 8} \mathrm{Q}^{4}+\frac{5}{9} \mathrm{P}^{2} \mathrm{Q}^{2}+\frac{5}{\mathrm{I} 2} \mathrm{P}^{3} \mathrm{Q}+\frac{8}{27} \mathrm{PQ}^{3}\right] \\
& \mathrm{P}_{49}(n+\mathrm{I})=\Delta_{5}(n) \cdot\left[\frac{2}{9} \mathrm{PQ}^{3}+\frac{\mathrm{I}}{3} \mathrm{P}^{2} \mathrm{Q}^{2}\right]+\Delta_{4}(n) \cdot\left[\frac{4}{8 \mathrm{I}} \mathrm{Q}^{4}+\frac{4}{9} \mathrm{PQ}^{3}+\frac{7}{\mathrm{r} 8} \mathrm{P}^{2} \mathrm{Q}^{2}\right] \\
& \mathrm{P}_{50}(n+\mathrm{I})=\Delta_{5}(n) \cdot\left[\frac{\mathrm{I}}{3} \mathrm{P}^{2} \mathrm{Q}^{2}\right]+\Delta_{4}(n) \cdot\left[\frac{\mathrm{I}}{9} \mathrm{P} \mathrm{Q}^{3}+\frac{7}{\mathrm{I} 8} \mathrm{P}^{2} \mathrm{Q}^{2}\right] \\
& \mathrm{P}_{51}(n+\mathrm{I})=\Delta_{5}(n) \cdot\left[\frac{3}{64} \mathrm{P}^{4}\right]+\Delta_{4}(n) \cdot\left[\frac{9}{\mathrm{I} 28} \mathrm{P}^{4}+\frac{\mathrm{I}}{\mathrm{I} 44} \mathrm{P}^{2} \mathrm{Q}^{2}+\frac{\mathrm{I}}{24} \mathrm{P}^{3} Q\right]+ \\
& +\Delta_{3}(n) \cdot\left[\frac{\mathrm{I}}{64^{8}} \mathrm{Q}^{4}+\frac{\mathrm{I}}{8} \mathrm{P}^{4}+\frac{\mathrm{I}}{\mathrm{I} 2} \mathrm{P}^{2} \mathrm{Q}^{2}+\frac{\mathrm{I}}{6} \mathrm{P}^{3} \mathrm{Q}+\frac{\mathrm{I}}{54} \mathrm{PQ}^{3}\right] \\
& +\Delta_{2}(n) \cdot\left[\frac{9}{\mathrm{I} 28} \mathrm{P}^{4}+\frac{\mathrm{I}}{32} \mathrm{P}^{2} \mathrm{Q}^{2}+\frac{3}{32} \mathrm{P}^{3} \mathrm{Q}\right] \\
& \mathrm{P}_{52}(n+\mathrm{I})=\Delta_{5}(n) \cdot\left[\frac{\mathrm{I}}{\mathrm{I} 2} \mathrm{P}^{2} \mathrm{Q}^{2}\right]+\Delta_{4}(n) \cdot\left[\frac{2}{27} \mathrm{PQ}^{3}+\frac{\mathrm{I}}{72} \mathrm{P}^{2} \mathrm{Q}^{2}\right] \\
& +\Delta_{3}(n) \cdot\left[\frac{8}{8 \mathrm{I}} \mathrm{Q}^{4}+\frac{8}{9} \mathrm{P}^{2} \mathrm{Q}^{2}+\frac{\mathrm{I} 6}{27} \mathrm{PQ}^{3}\right]+\Delta_{2}(n) \cdot\left[\frac{3}{8} \mathrm{P}^{2} \mathrm{Q}^{2}+\frac{\mathrm{I}}{4} \mathrm{PQ}^{3}\right] \\
& \mathrm{P}_{53}(n+\mathrm{r})=\Delta_{5}(n) \cdot\left[\frac{\mathrm{I}}{2 \mathrm{I} 6} \mathrm{Q}^{4}+\frac{3}{32} \mathrm{P}^{4}+\frac{\mathrm{I}}{24} \mathrm{P}^{2} \mathrm{Q}^{2}\right]+\Delta_{4}(n) \cdot\left[\frac{\mathrm{II}}{432} \mathrm{Q}^{4}+\frac{9}{64} \mathrm{P}^{4}+\frac{\mathrm{I} 9}{\mathrm{I} 44} \mathrm{P}^{2} \mathrm{Q}^{2}+\right. \\
& \left.+\frac{\mathrm{I}}{\mathrm{I} 2} \mathrm{P}^{3} \mathrm{Q}+\frac{\mathrm{I}}{27} \mathrm{PQ}^{3}\right]+\Delta_{3}(n) \cdot\left[\frac{\mathrm{I}}{4} \mathrm{Q}^{4}+\frac{\mathrm{I}}{4} \mathrm{P}^{4}+\frac{\mathrm{II}}{\mathrm{I} 8} \mathrm{P}^{2} \mathrm{Q}^{2}+\frac{\mathrm{I}}{3} \mathrm{P}^{3} \mathrm{Q}+\frac{\mathrm{I}}{3} \mathrm{PQ}^{3}\right] \\
& +\Delta_{2}(n) \cdot\left[\frac{\mathrm{I}}{\mathrm{I} 6} \mathrm{Q}^{4}+\frac{9}{64} \mathrm{P}^{4}+\frac{\mathrm{I}}{4} \mathrm{P}^{2} \mathrm{Q}^{2}+\frac{3}{\mathrm{I} 6} \mathrm{P}^{3} \mathrm{Q}+\frac{\mathrm{T}}{8} \mathrm{PQ}^{3}\right] \\
& \mathrm{P}_{54}(n+\mathrm{I})=\Delta_{5}(n) \cdot\left[\frac{\mathrm{I}}{\mathrm{I} 2} \mathrm{P}^{2} \mathrm{Q}^{2}\right] \\
& \mathrm{P}_{55}(n+\mathrm{I})=\Delta_{5}(n) \cdot\left[\frac{\mathrm{I}}{9} \mathrm{PQ}^{3}+\frac{\mathrm{I}}{6} \mathrm{P}^{2} \mathrm{Q}^{2}\right] \\
& \mathrm{P}_{58}(n+\mathrm{I})=\Delta_{5}(n) \cdot\left[\frac{\mathrm{I}}{6} \mathrm{P}^{2} \mathrm{Q}^{2}+\frac{\mathrm{I}}{4} \mathrm{P}^{8} \mathrm{Q}\right]+\Delta_{4}(n) \cdot\left[\frac{\mathrm{I}}{8 \mathrm{I}} \mathrm{Q}^{4}+\frac{23}{7^{2}} \mathrm{P}^{2} \mathrm{Q}^{2}+\frac{\mathrm{I}}{4} \mathrm{P}^{3} \mathrm{Q}+\frac{5}{54} \mathrm{PQ}^{3}\right] \\
& \mathrm{P}_{57}(n+\mathrm{I})=\Delta_{5}(n) \cdot\left[\frac{\mathrm{I}}{2} \mathrm{P}^{3} \mathrm{Q}+\frac{\mathrm{I}}{9} \mathrm{PQ}^{3}\right]+\Delta_{4}(n) \cdot\left[\frac{4}{8 \mathrm{I}} \mathrm{Q}^{4}+\frac{\mathrm{I}}{4} \mathrm{P}^{2} \mathrm{Q}^{2}+\frac{\mathrm{I}}{2} \mathrm{P}^{3} \mathrm{Q}+\frac{\mathrm{I} 3}{54} \mathrm{PQ}^{3}\right] \\
& \mathrm{P}_{58}(n+\mathrm{I})=\Delta_{5}(n) \cdot\left[\frac{2}{9} \mathrm{PQ}^{3}+\frac{\mathrm{I}}{3} \mathrm{P}^{2} \mathrm{Q}^{2}\right]+\Delta_{4}(n) \cdot\left[\frac{\mathrm{rO}}{8 \mathrm{I}} \mathrm{Q}^{4}+\frac{5}{9} \mathrm{P} Q^{3}+\frac{7}{\mathrm{I} 8} \mathrm{P}^{2} \mathrm{Q}^{2}\right] \\
& \mathrm{P}_{59}(n+\mathrm{I})=\Delta_{5}(n) \cdot\left[\frac{\mathrm{I}}{8} \mathrm{P}^{3} \mathrm{Q}\right]+\Delta_{4}(n) \cdot\left[\frac{5}{36} \mathrm{P}^{2} \mathrm{Q}^{2}+\frac{\mathrm{I} 3}{4^{8}} \mathrm{P}^{3} \mathrm{Q}+\frac{\mathrm{I}}{54} \mathrm{PQ}^{3}\right] \\
& +\Delta_{3}(n) \cdot\left[\frac{2}{8 \mathrm{I}} \mathrm{Q}^{4}+\frac{2}{3} \mathrm{P}^{2} \mathrm{Q}^{2}+\frac{2}{3} \mathrm{P}^{3} \mathrm{Q}+\frac{2}{9} \mathrm{PQ}^{3}\right]+\Delta_{2}(n) \cdot\left[\frac{7}{\mathrm{I} 6} \mathrm{P}^{2} \mathrm{Q}^{2}+\frac{3}{8} \mathrm{P}^{3} \mathrm{Q}+\frac{\mathrm{I}}{8} \mathrm{PQ}^{3}\right]
\end{aligned}
$$




$$
\begin{aligned}
& \mathrm{P}_{60}(n+\mathrm{I})=\Delta_{5}(n) \cdot\left[\frac{\mathrm{I}}{4} \mathrm{P}^{3} \mathrm{Q}+\frac{\mathrm{I}}{\mathrm{I} 8} \mathrm{PQ}^{3}\right]+\Delta_{4}(n) \cdot\left[\frac{4}{8 \mathrm{I}} \mathrm{Q}^{4}+\frac{5}{\mathrm{I} 8} \mathrm{P}^{2} \mathrm{Q}^{2}+\frac{\mathrm{I} 3}{24} \mathrm{P}^{3} \mathrm{Q}+\frac{29}{\mathrm{I} 08} \mathrm{PQ}^{3}\right] \\
& +\Delta_{3}(n) \cdot\left[\frac{4}{9} \mathrm{Q}^{4}+\frac{4}{3} \mathrm{P}^{2} \mathrm{Q}^{2}+\frac{4}{3} \mathrm{P}^{3} \mathrm{Q}+\frac{44}{27} \mathrm{PQ}^{3}\right] \\
& +\Delta_{2}(n) \cdot\left[\frac{\mathrm{r}}{4} \mathrm{Q}^{4}+\frac{7}{8} \mathrm{P}^{2} \mathrm{Q}^{2}+\frac{3}{4} \mathrm{P}^{3} \mathrm{Q}+\frac{3}{4} \mathrm{PQ}^{3}\right] \\
& \mathrm{P}_{61}(n+\mathrm{I})=\Delta_{5}(n) \cdot\left[\frac{\mathrm{I}}{\mathrm{I} 2} \mathrm{P}^{2} \mathrm{Q}^{2}+\frac{\mathrm{I}}{8} \mathrm{P}^{3} \mathrm{Q}\right]+\Delta_{4}(n) \cdot\left[\frac{5}{324} \mathrm{Q}^{4}+\frac{2}{9} \mathrm{P}^{2} \mathrm{Q}^{2}+\frac{\mathrm{I}}{8} \mathrm{P}^{3} \mathrm{Q}+\frac{\mathrm{II}}{\mathrm{IO} 8} \mathrm{PQ}^{3}\right] \\
& \mathrm{P}_{62}(n+\mathrm{I})=\Delta_{5}(n) \cdot\left[\frac{\mathrm{r}}{6} \mathrm{P}^{2} \mathrm{Q}^{2}\right]+\Delta_{4}(n) \cdot\left[\frac{2}{8 \mathrm{I}} \mathrm{Q}^{4}+\frac{4}{27} \mathrm{PQ}^{3}+\frac{5}{\mathrm{I} 8} \mathrm{P}^{2} \mathrm{Q}^{2}\right] \\
& \mathrm{P}_{63}(n+\mathrm{I})=\Delta_{5}(n) \cdot\left[\frac{3}{\mathrm{I} 6} \mathrm{P}^{4}+\frac{\mathrm{I}}{24} \mathrm{P}^{2} \mathrm{Q}^{2}\right]+\Delta_{4}(n) \cdot\left[\frac{\mathrm{I}}{8 \mathrm{I}} \mathrm{Q}^{4}+\frac{\mathrm{II}}{3^{2}} \mathrm{P}^{4}+\frac{\mathrm{II}}{{ }^{8}} \mathrm{P}^{2} \mathrm{Q}^{2}+\frac{\mathrm{I} 3}{4^{8}} \mathrm{P}^{3} \mathrm{Q}+\frac{\mathrm{I}}{\mathrm{I} 2} \mathrm{PQ}^{3}\right] \\
& +\Delta_{3}(n) \cdot\left[\frac{\mathrm{I}}{\mathrm{I} 8} \mathrm{Q}^{4}+\frac{\mathrm{I}}{2} \mathrm{P}^{4}+\frac{7}{9} \mathrm{P}^{2} \mathrm{Q}^{2}+\frac{2}{3} \mathrm{P}^{3} \mathrm{Q}+\frac{\mathrm{IO}}{27} \mathrm{PQ}^{3}\right] \\
& \div \Delta_{2}(n) \cdot\left[\frac{\mathrm{I}}{8} \mathrm{Q}^{4}+\frac{\mathrm{I} 5}{3^{2}} \mathrm{P}^{4}+\frac{7}{8} \mathrm{P}^{2} \mathrm{Q}^{2}+\frac{7}{8} \mathrm{P}^{3} \mathrm{Q}+\frac{\mathrm{I}}{2} \mathrm{PQ}^{3}\right] \\
& \mathrm{P}_{64}(n+\mathrm{I})=\Delta_{5}(n) \cdot\left[\frac{\mathrm{I}}{\mathrm{I} 2} \mathrm{P}^{2} \mathrm{Q}^{2}\right]+\Delta_{4}(n) \cdot\left[\frac{2}{8 \mathrm{I}} \mathrm{Q}^{4}+\frac{4}{27} \mathrm{PQ}^{3}+\frac{7}{24} \mathrm{P}^{2} \mathrm{Q}^{2}\right] \\
& +\Delta_{3}(n) \cdot\left[\frac{8}{8 \mathrm{I}} \mathrm{Q}^{4}+\frac{8}{9} \mathrm{P}^{2} \mathrm{Q}^{2}+\frac{\mathrm{I} 6}{27} \mathrm{PQ}^{3}\right]+\Delta_{2}(n) \cdot\left[\frac{\mathrm{r}}{4} \mathrm{Q}^{4}+\frac{5}{8} \mathrm{P}^{2} \mathrm{Q}^{2}+\frac{3}{4} \mathrm{PQ}^{3}\right] \\
& \mathrm{P}_{65}(n+\mathrm{I})=\Delta_{5}(n) \cdot\left[\frac{\mathrm{I}}{8} \mathrm{P}^{3} \mathrm{Q}\right]+\Delta_{4}(n) \cdot\left[\frac{\mathrm{I}}{8 \mathrm{r}} \mathrm{Q}^{4}+\frac{\mathrm{I}}{3} \mathrm{P}^{2} \mathrm{Q}^{2}+\frac{\mathrm{I} 9}{4^{8}} \mathrm{P}^{3} \mathrm{Q}+\frac{\mathrm{I}}{9} \mathrm{P} \mathrm{Q}^{3}\right] \\
& +\Delta_{3}(n) \cdot\left[\frac{2}{8 \mathrm{r}} \mathrm{Q}^{4}+\frac{2}{3} \mathrm{P}^{2} \mathrm{Q}^{2}+\frac{2}{3} \mathrm{P}^{3} \mathrm{Q}+\frac{2}{9} \mathrm{PQ}^{3}\right] \\
& +\Delta_{2}(n) \cdot\left[\frac{\mathrm{r}}{4} \mathrm{Q}^{4}+\frac{27}{\mathrm{r} 6} \mathrm{P}^{2} \mathrm{Q}^{2}+\frac{7}{8} \mathrm{P}^{3} \mathrm{Q}+\frac{9}{8} \mathrm{PQ}^{3}\right] \\
& \mathrm{P}_{66}(n+\mathrm{I})=\Delta_{5}(n) \cdot\left[\frac{\mathrm{I}}{64} \mathrm{P}^{4}\right]+\Delta_{4}(n) \cdot\left[\frac{\mathrm{I}}{\mathrm{I} 296} \mathrm{Q}^{4}+\frac{9}{\mathrm{I} 28} \mathrm{P}^{4}+\frac{\mathrm{I}}{24} \mathrm{P}^{2} \mathrm{Q}^{2}+\frac{\mathrm{I}}{\mathrm{I} 2} \mathrm{P}^{3} \mathrm{Q}+\frac{\mathrm{I}}{\mathrm{I} 08} \mathrm{PQ}^{3}\right] \\
& +\Delta_{3}(n) \cdot\left[\frac{\mathrm{I}}{648} \mathrm{Q}^{4}+\frac{\mathrm{I}}{8} \mathrm{P}^{4}+\frac{\mathrm{I}}{\mathrm{I} 2} \mathrm{P}^{2} \mathrm{Q}^{2}+\frac{\mathrm{I}}{6} \mathrm{P}^{3} \mathrm{Q}+\frac{\mathrm{I}}{54} \mathrm{PQ}^{3}\right] \\
& +\Delta_{2}(n) \cdot\left[\frac{\mathrm{I}}{\mathrm{I} 6} \mathrm{Q}^{4}+\frac{4 \mathrm{I}}{\mathrm{I} 28} \mathrm{P}^{4}+\frac{27}{32} \mathrm{P}^{2} \mathrm{Q}^{2}+\frac{27}{32} \mathrm{P}^{3} \mathrm{Q}+\frac{3}{8} \mathrm{PQ}^{3}\right]+\Delta_{1}(n) \text {. }
\end{aligned}
$$

Ces résultats sont fondamentaux pour l'étude de la covariance entre les 2 descendants $\mathrm{I}$ et $\mathrm{J}$ (appartenant tous les 2 à la génération $(n+\mathrm{I})$ ) de $\mathrm{H}_{n}$.

Par exemple, lorsqu'on suppose les effets de dominance de tous ordres négligeables, la covariance génotypique se réduit à :

$$
\begin{aligned}
\operatorname{cov}(\mathrm{I}, \mathrm{J})_{n+1} & =4 \Delta_{5}(n)+6 \Delta_{4}(n)+8 \Delta_{3}(n)+\text { Io } \Delta_{2}(n)+\mathrm{I} 6 \Delta_{1}(n) \\
& =\mathrm{I} 6 \varphi_{n}^{(111)} \\
& =\mathrm{I}+3 f_{n}^{(2)}
\end{aligned}
$$

$\varphi\left(\frac{111}{n}\right)$ est la probabilité pour qu'un gène pris dans $\mathrm{I}$ et un gène pris dans $\mathrm{J}$ soient identiques. Ce coefficient de parenté dépend du déroulement de la méiose (car les $\Delta_{k}^{(n)}$ sont des fonctions de $\mathrm{P}$ ). Le rapport de corrélation génotypique $r(\mathrm{I}, \mathrm{J})_{n+1}$ est alors égal au rapport des valeurs prises par le coefficient de parenté $\varphi(\underline{I} \mid \mathrm{I})$ aux instants $n$ et $n+\mathrm{I}$.

$$
r(\mathrm{I}, \mathrm{J})_{n+1}=\frac{\varphi_{n}^{\left(\frac{111}{1}\right)}}{\varphi_{n+1}^{\left(\frac{1 ! 1}{n}\right)}}=\frac{\mathrm{I}+3 f_{n}^{(2)}}{\mathrm{I}+3 f_{n+1}^{(2)}}
$$


Il est à remarquer que ce résultat est plus général que celui établi par KEMPTHORNE (I957) dans le cas d'une méiose chromosomique, bien que la formulation soit la même.

Si maintenant on ne néglige que les effets de dominance d'ordre 3 et 4 , l'expression de la covariance génotypique se complique, puisque nous continuons à considérer la méiose chromatidique. Nous obtenons la formule suivante $\mathrm{n}^{0} 2$ :

$$
\begin{aligned}
& \operatorname{cov}(\mathrm{I}, \mathrm{J})_{n+1}=\left[4 \Delta_{5}(n)+6 \Delta_{4}(n)+8 \Delta_{3}(n)+\text { Io } \Delta_{2}(n)+\mathrm{I} 6 \Delta_{1}(n)\right] . \mathrm{E}\left(Z^{2}\right) \\
& +\left[2 \Delta_{5}(n)\left(\mathrm{Q}^{4}+3 \mathrm{P}^{4}+{ }_{\mathrm{I} 2} \mathrm{P}^{2} \mathrm{Q}^{2}+\mathrm{IoP}^{3} \mathrm{Q}+6 \mathrm{PQ}^{3}\right)+\right. \\
& +\Delta_{4}(n)\left(\frac{I 9}{3} Q^{4}+\mathrm{IIP}^{4}+5_{22} \mathrm{P}^{2} \mathrm{Q}^{2}+\frac{\mathrm{II} 8}{3} \mathrm{P}^{3} \mathrm{Q}+3^{0} \mathrm{PQ}^{3}\right) \\
& +\Delta_{3}(n)\left(\frac{32}{3} \mathrm{Q}^{4}+\mathrm{I} 6 \mathrm{P}^{4}+80 \mathrm{P}^{2} \mathrm{Q}^{2}+\frac{\mathrm{I} 76}{3} \mathrm{P}^{3} \mathrm{Q}+48 \mathrm{PQ}^{3}\right) \\
& +4 \Delta_{2}(n)\left(5 \mathrm{Q}^{4}+6 \mathrm{P}^{4}+33 \mathrm{P}^{2} \mathrm{Q}^{2}+23 \mathrm{P}^{3} \mathrm{Q}+2 \mathrm{IPQ}^{3}\right) \\
& \left.+48 \Delta_{1}(n)\right] \cdot \mathrm{E}\left(Z_{i} \mathrm{D}_{i i}\right) \\
& +\left[\Delta_{5}(n)\left(\frac{\mathrm{I}}{4} \mathrm{Q}^{4}+\frac{9}{4} \mathrm{P}^{4}+\frac{\mathrm{II}}{2} \mathrm{P}^{2} \mathrm{Q}^{2}+6 \mathrm{P}^{3} \mathrm{Q}+2 \mathrm{PQ}^{3}\right)\right. \\
& +\Delta_{4}(n)\left(\frac{\mathrm{r} 37}{72} \mathrm{Q}^{4}+\frac{4 \mathrm{I}}{8} \mathrm{P}^{4}+\frac{69 \mathrm{I}}{36} \mathrm{P}^{2} \mathrm{Q}^{2}+\frac{49}{3} \mathrm{P}^{3} \mathrm{Q}+\frac{89}{9} \mathrm{PQ}^{3}\right) \\
& +\Delta_{3}(n)\left(\frac{32}{9} \mathrm{Q}^{4}+8 \mathrm{P}^{4}+\frac{296}{9} \mathrm{P}^{2} \mathrm{Q}^{2}+\frac{8 \mathrm{o}}{3} \mathrm{P}^{3} \mathrm{Q}+\frac{\mathrm{I} 6 \mathrm{o}}{9} \mathrm{PQ}^{3}\right) \\
& +\Delta_{2}(n)\left(\frac{\mathrm{I} 3}{8} \mathrm{Q}^{4}+\frac{\mathrm{II} 7}{8} \mathrm{P}^{4}+\frac{299}{4} \mathrm{P}^{2} \mathrm{Q}^{2}+54 \mathrm{P}^{3} \mathrm{Q}+46 \mathrm{PQ}^{3}\right) \\
& \left.+36 \Delta_{1}(n)\right] \cdot \mathrm{E}\left(\mathrm{D}_{i i}{ }^{2}\right) \\
& +\left(\frac{\mathrm{I}}{6} \Delta_{5}(n)+\frac{\mathrm{I}}{4} \Delta_{4}(n)+\frac{4}{9} \Delta_{3}(n)+\frac{\mathrm{I}}{4} \Delta_{2}(n)\right) . \\
& .\left(25 \mathrm{Q}^{4}+9 \mathrm{P}^{4}+94 \mathrm{P}^{2} \mathrm{Q}^{2}+48 \mathrm{P}^{3} \mathrm{Q}+80 \mathrm{PQ}^{3}\right) \cdot \mathrm{E}\left(\mathrm{D}_{i j}{ }^{2}\right) \\
& +\left[\Delta_{5}(n)\left(\frac{3}{4} \mathrm{Q}^{4}+\frac{27}{4} \mathrm{P}^{4}+\frac{33}{2} \mathrm{P}^{2} \mathrm{Q}^{2}+\mathrm{I} 8 \mathrm{P}^{3} \mathrm{Q}+6 \mathrm{PQ}^{3}\right)\right. \\
& +\Delta_{4}(n)\left(\frac{35}{24} \mathrm{Q}^{4}+\frac{57}{8} \mathrm{P}^{4}+\frac{265}{\mathrm{I} 2} \mathrm{P}^{2} \mathrm{Q}^{2}+2 \mathrm{IP}^{3} \mathrm{Q}+\frac{29}{3} \mathrm{PQ}^{3}\right) \\
& +\Delta_{3}(n)\left(\frac{32}{9} \mathrm{Q}^{4}+8 \mathrm{P}^{4}+\frac{296}{9} \mathrm{P}^{2} \mathrm{Q}^{2}+\frac{8 \mathrm{o}}{3} \mathrm{P}^{3} \mathrm{Q}+\frac{\mathrm{r6o}}{9} \mathrm{PQ}^{3}\right) \\
& \left.+\Delta_{2}(n)\left(\frac{\mathrm{r} 3}{3} \mathrm{Q}^{4}+\frac{45}{8} \mathrm{P}^{4}+\frac{83}{4} \mathrm{P}^{2} \mathrm{Q}^{2}+\mathrm{r} 8 \mathrm{P}^{3} \mathrm{Q}+\mathrm{ro} \mathrm{PQ}^{3}\right)\right] \\
& {\left[\mathrm{E}\left(\mathrm{D}_{i i}\right)\right]^{2}-\left[\Delta_{1}(n)+\frac{\mathrm{x}}{2} \Delta_{2}(n)+\frac{\mathrm{I}}{3} \Delta_{3}(n)+\frac{\mathrm{I}}{6} \Delta_{4}(n)\right]^{2} \cdot\left[\mathrm{E}\left(\mathrm{D}_{i i}\right)\right]^{2}}
\end{aligned}
$$

Si on ne néglige aucun des effets de dominance, on écrit de façon analogue la covariance génotypique en utilisant la formule générale donnée par Jacqueline BOUFFETTE (I966). 
Pour une méiose chromosomique, il suffit de faire dans tous les résultats $P=0$; cette hypothèse, parfois justifiée, simplifie nos résultats qui alors, peuvent toujours être considérés comme des résultats approchés.

\title{
CONCLUSION
}

Les résultats que nous avons obtenus, aussi bien l'expression de la variance génotypique, que celle de la covariance génotypique reflètent la complexité de l'étude des tétraploïdes. Nous pensons cependant avoir, pour la première fois, tenu compte, pour l'établissement des formules théoriques (I) et (2), des 2 faits suivants :

- l'existence des effets de dominance possibles entre 2, 3 et 4 gènes homologues,

- la possibilité d'obtenir des gamètes pouvant porter des gènes frères ou des gènes non frères.

Rę̧u pour publication en octobre 1968.

\author{
SUMMARY \\ A PRIORI GENOTYPIC VARIANCE AND COVARIANCE \\ IN A TETRAPLOID POPULATION UNDER SELF-FERTILIZATION
}

For a tetraploid individual taken at a given generation in a self-fertilized population, the theoretical constitution of the a priori genotypic variance is estimated by the previously presented formula developed by Jacqueline BoufFeTte for any two tetraploid zygotes and by the calculation of the coefficients of identity restricted to order 4 , rank 1 .

The coefficients of restricted identity to order 8, rank 2, attached to two zygotes taken at random from among the descendants of a similar individual in generation " $n$ " are expressed linearly as a function of the coefficients of identity restricted to order 4 , rank 1 . Using once more the results of Jacqueline BouffetTE, it is possible to derive the most general theoretical expression of the a priori genotypic covariance among two full sib tetraploid individuals. This is shown for two cases; first under the assumption of negligible dominance at all levels, and second under the assumption of negligible effects of dominance involving 3 or 4 homologous genes.

The expressions obtained are new : they take into account the possible dominance effects and the complexity of meiosis in tetraploids, because the various coefficients which feature in the expressions are functions of the probability that a gamete carries two "sibling-genes ", that is, copies of a single gene present in the ancestor.

\section{RÉFÉRENCES BIBLIOGRAPHIQUES}

Bouffetre A. R., I966. Contribution mathématique à la génétique des tétraploïdes. Thèse $3^{\mathrm{e}} c y c l e$, Lyon.

Boufferte Jacqueline, 1966. Expression de la covariance génotypique chez les tétraploïdes. Thèse $3^{\mathrm{e}}$ cycle, Lyon.

Gallars A., 1967. Modèle pour l'étude des effets génétiques. Ann. Amélior. Plantes, 17, $229-242$.

Girlors M., 1965. Relation d'identité en génétique. Ann. Inst. Henri-Poincaré, 2, r-94.

KeMPthoRNe O., x957. An introduction to genetic statistics. J. Wiley and Sons, New-York.

Malecot G., 1948. Les mathématiques de l'hérédité, Masson, Paris. 